\title{
Sign change of the Soret coefficient of poly(ethylene oxide) in water/ethanol mixtures observed by thermal diffusion forced Rayleigh scattering
}

\author{
Rio Kita \\ Max-Planck-Institut für Polymerforschung, Ackermannweg 10, D-55128 Mainz, Germany \\ Simone Wiegand ${ }^{\text {a) }}$ \\ Max-Planck-Institut für Polymerforschung, Ackermannweg 10, D-55128 Mainz, Germany \\ and Forschungszentrum Jülich GmbH, IFF_Weiche Materie, D-52428 Jülich, Germany \\ Jutta Luettmer-Strathmann \\ Department of Physics, The University of Akron, Akron, Ohio 44325-4001
}

(Received 24 December 2003; accepted 18 May 2004)

\begin{abstract}
Soret coefficients of the ternary system of poly(ethylene oxide) in mixed water/ethanol solvent were measured over a wide solvent composition range by means of thermal diffusion forced Rayleigh scattering. The Soret coefficient $S_{\mathrm{T}}$ of the polymer was found to change sign as the water content of the solvent increases with the sign change taking place at a water mass fraction of 0.83 at a temperature of $22^{\circ} \mathrm{C}$. For high water concentrations, the value of $S_{\mathrm{T}}$ of poly (ethylene oxide) is positive, i.e., the polymer migrates to the cooler regions of the fluid, as is typical for polymers in good solvents. For low water content, on the other hand, the Soret coefficient of the polymer is negative, i.e., the polymer migrates to the warmer regions of the fluid. Measurements for two different polymer concentrations showed a larger magnitude of the Soret coefficient for the smaller polymer concentration. The temperature dependence of the Soret coefficient was investigated for water-rich polymer solutions and revealed a sign change from negative to positive as the temperature is increased. Thermodiffusion experiments were also performed on the binary mixture water/ethanol. For the binary mixtures, the Soret coefficient of water was observed to change sign at a water mass fraction of 0.71 . This is in agreement with experimental results from the literature. Our results show that specific interactions (hydrogen bonds) between solvent molecules and between polymer and solvent molecules play an important role in thermodiffusion for this system. (C) 2004 American Institute of Physics. [DOI: 10.1063/1.1771631]
\end{abstract}

\section{INTRODUCTION}

The presence of a temperature gradient in a fluid mixture generally induces mass flows, which create concentration gradients in the mixture. This process is known as the Ludwig-Soret effect. ${ }^{1,2}$ In a binary mixture, the size of the effect is characterized by the Soret coefficient, which relates the gradient of the concentration to the gradient of the temperature in the steady state

$$
S_{\mathrm{T}}=-\frac{1}{c_{0}\left(1-c_{0}\right)} \frac{\boldsymbol{\nabla} c}{\nabla T} .
$$

Here $T$ is the temperature, $c$ is the mass fraction of component $1, c_{0}$ its equilibrium value, and $\boldsymbol{\nabla}$ indicates the gradient. The Soret coefficient defined by Eq. (1) is properly called the Soret coefficient of component $1 .^{3,4}$ It has a positive sign when component 1 migrates to the cold side and a negative sign when component 1 migrates to the warm side. In a binary mixture, the Soret coefficient can be expressed as $S_{\mathrm{T}}$ $=\left(D_{\mathrm{T}} / D\right)$, where $D_{\mathrm{T}}$ is the thermal diffusion coefficient and $D$ is the ordinary translational diffusion coefficient. ${ }^{5}$

\footnotetext{
${ }^{a)}$ Electronic mail: s.wiegand@fz-juelich.de; http://www.fz-juelich.de/iff/ personen/S.Wiegand/
}

Thermal diffusion in gas mixtures is well described by the Chapman-Enskog theory. ${ }^{6}$ For very simple gaseous systems it is even possible to calculate the thermal diffusion coefficient by $a b$ initio methods. ${ }^{7,8}$ In gas mixtures, where the interactions between molecules are dominated by hard sphere repulsion, it is predicted and experimentally observed that the heavier component migrates to the cold side (positive $S_{\mathrm{T}}$ ). ${ }^{6}$ Analogous results are also typically observed for liquid mixtures, such as polymer solutions and colloidal suspensions, where the solute is much heavier than the solvent molecules. For instance, the well studied system of polystyrene (PS) in a good solvent shows a positive $S_{\mathrm{T}}$ value of PS. ${ }^{9-13}$ However, in a few cases, negative $S_{\mathrm{T}}$ values have been found for the heavier component in liquid mixtures of components with large differences in molecular mass. Giglio and Vendramini reported a negative Soret coefficient for poly(vinyl alcohol) in water. ${ }^{14}$ Recently, we found a negative $S_{\mathrm{T}}$ for poly(ethylene oxide) (PEO) in water/ethanol mixtures with high ethanol content, ${ }^{15,16}$ while we observed a positive $S_{\mathrm{T}}$ in pure water. Iacopini and Piazza ${ }^{17}$ also found a sign change of the Soret coefficient in recent thermophoresis experiments on protein solutions.

Sign changes of the Soret coefficient are well known for a number of small-molecules fluid mixtures. ${ }^{18}$ Mixtures of 
water and ethanol, for instance, have been studied carefully by several authors. ${ }^{19-21}$ In a mixture with high ethanol content $S_{\mathrm{T}}$ of ethanol is negative (ethanol molecules migrate to the hot side) whereas at low ethanol content, $S_{\mathrm{T}}$ of ethanol is positive (ethanol molecules migrate to the cold side). Debuschewitz and Köhler investigated benzene/cyclohexane mixtures with different isotopes and found that mass differences yield a constant contribution to the Soret coefficient while chemical differences are responsible for sign changes of the Soret coefficients. ${ }^{22}$ Recently, the first molecular dynamics simulations of the Soret effect in aqueous solutions were performed that agree quantitatively with experimental data. ${ }^{23,24}$

Thermal diffusion in liquid mixtures is not well understood theoretically. ${ }^{25}$ For liquids with specific interactions such as hydrogen bonds it is often not even possible to predict the sign of $S_{\mathrm{T}}$ correctly. Prigogine et al. ${ }^{18}$ argued that entropic as well as energetic effects have to be taken into account in order to understand thermodiffusion in mixtures of associating fluids. For colloids in solution, Bringuier and Bourdon derived expressions for Soret coefficients using the kinetic theory of Brownian motion. ${ }^{26}$ They find that particle movement towards low-temperature regions is strengthened or reversed by a temperature dependent interaction potential. Dhont $^{27,28}$ recently developed a microscopic approach to thermodiffusion of colloids in solution including hydrodynamic interactions. Dhont relates the thermal diffusion coefficient $D_{\mathrm{T}}$ to an effective potential for interactions between the colloidal particle. He shows that the thermal diffusion coefficient $D_{\mathrm{T}}$ has a temperature independent contribution, which is always positive, and a temperature-dependent contribution, which depends on the interparticle potential and may change the sign of the thermodiffusion coefficient. These concepts have yet to be extended to thermodiffusion in polymer solutions, where single-particle properties and polymer-solvent interactions become important.

Because of its importance in industrial applications, studies of the Ludwig-Soret effect in mixtures of three or more components have also been carried out. ${ }^{29-34}$ The determination of Soret coefficients and mass diffusion constants in those mixtures is still subject to discussion. Even for ternary mixtures, different definitions of the thermal diffusion coefficient exist. ${ }^{31-33}$ In general, care has to be taken with the choice of reference frame and one should recover the standard definition of the coefficients in the binary limits. In the case of a ternary system of a polymer with two solvents, two processes are expected to be significant in determining the thermodiffusion of the polymer. In response to the temperature gradient, a gradient in the solvent composition builds up rapidly. The polymer then migrates in response to both the temperature and the solvent composition gradients. Thus the composition of the solvent mixture plays a key role in the thermal diffusion behavior of the polymer. In fact, a different $S_{\mathrm{T}}$ value of PS was observed for PS in mixed solvents corresponding to good and a poor solvent conditions, respectively. This implies that, in addition to the temperature gradient, the concentration gradient of the solvent mixture affects the concentration gradient of PS. ${ }^{34}$

We recently reported thermal diffusion data for the ter- nary system of PEO in water/ethanol mixtures ${ }^{16}$ obtained by a holographic grating method, called thermal diffusion forced Rayleigh scattering (TDFRS). These data showed, for the first time, a change in sign of the Soret coefficient of a polymer in solution. In order to understand these results, a two-chamber lattice model for thermodiffusion in liquid mixtures was developed. ${ }^{15,35,36}$ The model qualitatively agrees with the experimental observations that the Soret coefficient of PEO is positive for water-rich solutions and changes sign as the ethanol content of the solvent increases. The model also predicts a sign change of the polymer's $S_{\mathrm{T}}$ value with changing temperature. We include here a comparison between experimental and theoretical results for the Soret coefficient of PEO in water/ethanol mixtures and for the Soret coefficient of water in binary mixtures of ethanol and water.

In the first experimental studies on the PEO/water/ ethanol system, measurements were performed on PEO in pure water and in mixed solvent of fairly high ethanol concentrations. ${ }^{16}$ The reason for this restriction was that the effect of the solvent concentration gradient became significant in the intermediate concentration range, which made the data analysis difficult. Since the determination of the Soret coefficient of PEO in the entire water/ethanol composition range is expected to provide insight into the underlying mechanisms, we have refined our data analysis so that we are now able to investigate thermal diffusion for all solvent compositions. In this work, we determine two Soret coefficients and two mass diffusion coefficients from each TDFRS measurement on PEO in mixed solvent. One pair of coefficients is associated with the solvent water/ethanol, the other is associated with the polymer in the mixed solvent. We present experimental TDFRS data for the Soret coefficient of PEO in water/ethanol mixtures for solvent compositions in the range of $0.05<w<1$, where $w$ is the mass fraction of water and for polymer concentrations of $5 \mathrm{~g} / \mathrm{L}$ and $1 \mathrm{~g} / \mathrm{L}$ of PEO, respectively. We also performed temperature dependent measurements of the Soret coefficient of the polymer and find that an increase in temperature can change the sign of $S_{\mathrm{T}}$. In order to further investigate thermodiffusion of the solvents, we performed TDFRS experiments on the binary system water/ ethanol. We compare the results of the binary system with those attributed to the solvent in the ternary systems PEO/ water/ethanol and find good agreement between the corresponding Soret coefficients.

The paper is organized as follows: in the following section, Sec. II, we present the working equations for binary and ternary mixtures that are used to analyze the TDFRS experiments. In Sec. III we briefly describe sample preparation and experimental apparatus. Experimental results for refractive index increments of the binary and ternary mixtures are important for the evaluation of the TDFRS experiments and are presented in Sec. IV A. Results on thermodiffusion in the binary system water/ethanol and the ternary system PEO/ water/ethanol are presented and discussed in Secs. IV B and IV C, respectively. We summarize our conclusions in Sec. V.

\section{INTERPRETATION OF THE TDFRS SIGNAL}

In a TDFRS experiment, an intensity grating is created by the interference of two laser beams. A trace amount of dye 
in the solution absorbs energy and converts the intensity grating into a temperature grating. The spatial variation of the temperature induces mass flows through the LudwigSoret effect. Both temperature and composition modulation contribute to the spatial modulation of the index of refraction, which is detected by Bragg refraction of an additional laser. In the Appendix we derive expressions for the normalized heterodyne signal intensity $\zeta_{\text {het }}$ of the read out laser. For a binary mixture,

$$
\begin{aligned}
\zeta_{\text {het }}= & 1-e^{-t / \tau_{\mathrm{th}}}-\frac{\left(\partial n / \partial c_{1}\right)_{P, T}}{(\partial n / \partial T)_{P, c_{1}}} c_{10}\left(1-c_{10}\right) \\
& \times S_{\mathrm{T}}\left(1-e^{-q^{2} D t}\right),
\end{aligned}
$$

where $\tau_{\text {th }}$ is the time constant of the temperature grating, $n$ is the index of refraction, $P$ is the pressure, $c_{1}$ is the mass fraction of component $1, c_{10}$ its equilibrium value, and where $q$ is the wave number of the intensity grating. For a ternary mixture,

$$
\begin{aligned}
\zeta_{\text {het }}= & 1-e^{-t / \tau_{\text {th }}}-\frac{\left(\partial n / \partial c_{1}\right)_{P, T, c_{2}}}{(\partial n / \partial T)_{P, c_{1}, c_{2}}} c_{10}\left(1-c_{10}\right) \\
& \times S_{1 T}\left(1-e^{\left.-q^{2} D_{11} t\right)}-\frac{\left(\partial n / \partial c_{2}\right)_{P, T, c_{1}}}{(\partial n / \partial T)_{P, c_{1}, c_{2}}} c_{20}\left(1-c_{20}\right)\right. \\
& \times S_{2 T}\left(1-e^{-q^{2} D_{22} t}\right)
\end{aligned}
$$

where $c_{k}$ and $c_{k 0}$ are the mass fraction of component $k$ and its equilibrium value, respectively, and where $D_{11}$ and $D_{22}$ are the diagonal elements of the matrix of diffusion coefficients. In analogy to binary mixtures, cf. Eq. (1), we have defined Soret coefficients $S_{k}$ for each component from the ratio of mass fraction and temperature gradients in the steady state, where the mass flows vanish

$$
S_{k T} \equiv-\frac{1}{c_{k 0}\left(1-c_{k 0}\right)} \frac{\nabla c_{k}}{\nabla T} .
$$

The expressions for the normalized heterodyne signal, Eqs. (2) and (3), for binary and ternary mixtures, respectively, show that a single diffusive process with time constant $\tau=1 /\left(q^{2} D\right)$ is expected for binary mixtures, while two diffusive processes with time constants $\tau_{1}=1 /\left(q^{2} D_{11}\right)$ and $\tau_{2}$ $=1 /\left(q^{2} D_{22}\right)$ are expected for the ternary system of a polymer in mixed solvent. Our experiments on PEO in water/ ethanol do indeed show a two-mode decay of the signal (see Sec. IV C) with time constants on the order of $10^{-3} \mathrm{~s}$ for the faster process, associated with solvent diffusion, and $10^{-1} \mathrm{~s}$ for the slower process, associated with diffusion of the polymer. Hence, the typical time constants of the diffusive processes are well separated on the time scale of the experiment.

The amplitudes of the decay modes of the normalized heterodyne signal are a product of contrast factors (combinations of derivatives of the index of refraction) and Soret coefficients. In Sec. IV A, we describe the determination of contrast factors from separate measurements. In order to ease notation, we introduce the following definitions:

(1) binary system

$$
\left(\frac{\partial n}{\partial T}\right)_{0} \equiv\left(\frac{\partial n}{\partial T}\right)_{P, w}, \quad\left(\frac{\partial n}{\partial w}\right)_{0} \equiv\left(\frac{\partial n}{\partial w}\right)_{P, T}
$$

(2) ternary system

$$
\begin{aligned}
& \left(\frac{\partial n}{\partial T}\right)_{1} \equiv\left(\frac{\partial n}{\partial T}\right)_{P, w, c}, \quad\left(\frac{\partial n}{\partial w}\right)_{1} \equiv\left(\frac{\partial n}{\partial w}\right)_{P, T, c}, \\
& \left(\frac{\partial n}{\partial c}\right)_{1} \equiv\left(\frac{\partial n}{\partial c}\right)_{P, T, w},
\end{aligned}
$$

where $w$ and $c$ are the mass fractions of water and PEO, respectively. In practice, we approximate $(\partial n / \partial w)_{1}$ by $(\partial n / \partial w)_{0}$.

\section{EXPERIMENT}

The experimental setup for TDFRS has been described in detail in Ref. 37. In brief, the interference grating is written by an argon ion laser operating at the wavelength of $\lambda$ $=488 \mathrm{~nm}$. The grating is read out by a He-Ne laser at $\lambda$ $=632.8 \mathrm{~nm}$. In the TDFRS measurements a rectangular quartz cell with a path length of $0.2 \mathrm{~mm}$ (Hellma) was used. The intensity of the diffracted beam was measured by a photomultiplier. A mirror mounted on a piezocrystal is used for phase shifting and stabilization to obtain the heterodyne signal. The TDFRS measurements were carried out at a temperature of $22.00^{\circ} \mathrm{C}$, where the temperature of the sample cell was controlled by circulating water from a thermostat with an uncertainty of $0.01{ }^{\circ} \mathrm{C}$.

PEO was polymerized from ethylene oxide in tetrahydrofuran by anionic polymerization at $60^{\circ} \mathrm{C}$. The weightand number-averaged molecular weight of PEO are $M_{\mathrm{w}}$ $=2.65 \times 10^{5} \mathrm{~g} / \mathrm{mol}$ and $M_{\mathrm{n}}=2.36 \times 10^{5} \mathrm{~g} / \mathrm{mol}$, respectively, determined by GPC. The index of molecular weight distribution is $M_{\mathrm{w}} / M_{\mathrm{n}}=1.1$. Deionized water (milli-Q) and ethanol (Riedel-de Haën, HPLC-grade) were used as solvent. The water content of ethanol was $0.11 \mathrm{wt} \%$ determined by Karl Fisher titration. A detailed description of the sample preparation is presented in Ref. 16. In this study, water/ethanol mixtures of different compositions and solutions of $5 \mathrm{~g} / \mathrm{L}$ PEO in such mixtures were prepared with trace amount of the dye Basantol Yellow 215 (BASF). The sample solutions were filtered directly into the cell through $0.22 \mu \mathrm{m}$ membrane filter (Millipore).

The absorption spectra of the dye in solutions of PEO/ water/ethanol and of water/ethanol, respectively, agree well over the entire composition range of water/ethanol solutions; an example is shown in Fig. 1. The peak at $460 \mathrm{~nm}$ indicates strong absorption of light at the wavelength $488 \mathrm{~nm}$ of the argon ion laser used to write the intensity grating. Absorption at $632.8 \mathrm{~nm}$ is negligible, which implies no contribution of the dye to the signal read by the He-Ne laser. In our earlier work on mixtures with high ethanol content we used quinizarin as a dye. ${ }^{15,16}$ The results for the mass and thermal diffusion coefficients of the present study for ethanol-rich solutions agree well with the previous results, implying that the results are independent of the choice of dye. 


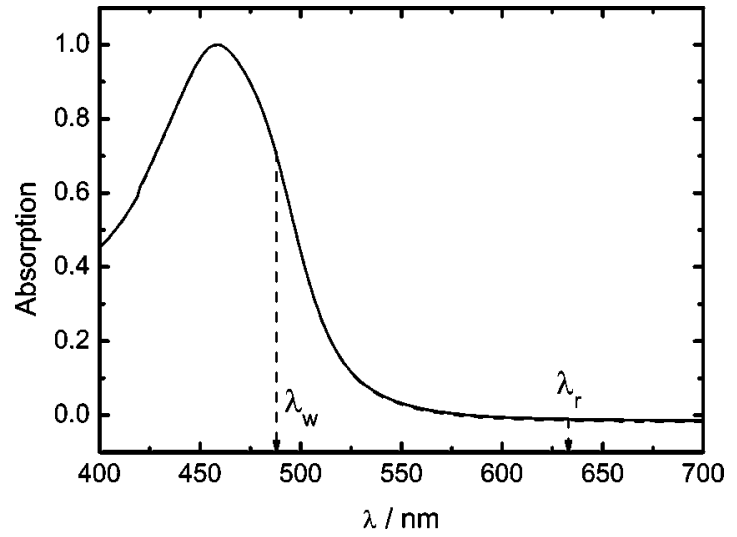

FIG. 1. Absorption spectra of Basantol Yellow 215 dissolved in PEO/water/ ethanol and in water/ethanol, respectively. The solid curve corresponds to a water/ethanol mixture with a mass fraction $w=0.2$ of water. The dashed curve corresponds to a solution of $5 \mathrm{~g} / \mathrm{L}$ PEO in water/ethanol $(w=0.2)$. The two curves coincide with each other.

\section{RESULTS AND DISCUSSION}

\section{A. Contrast factors}

Refractive index increments with respect to the concentration and the temperature for the systems of $\mathrm{PEO} / \mathrm{water} /$ ethanol and water/ethanol are shown in Figs. 2 and 3, respectively. $(\partial n / \partial c)_{1}$ was measured at room temperature using a scanning Michelson interferometer operating at a wavelength of $632.8 \mathrm{~nm}^{38}(\partial n / \partial T)_{1}$ of $\mathrm{PEO} /$ water/ethanol was measured at $632.8 \mathrm{~nm}$ in the temperature range $293.65-296.65$ $\mathrm{K} .{ }^{38}$ Figure 2 presents $(\partial n / \partial c)_{1}$ and $(\partial n / \partial T)_{1}$ of $5 \mathrm{~g} / \mathrm{L}$ PEO in the solvent mixture as a function of the mass fraction of water for the water/ethanol solvent. The deviation of $(\partial n / \partial c)_{1}$ from the fitted function (solid line) is less than $1.5 \%$. Figure 3 shows $(\partial n / \partial w)_{0}$ and $(\partial n / \partial T)_{0}$ of water/ ethanol mixtures as a function of mass fraction of water $w$.

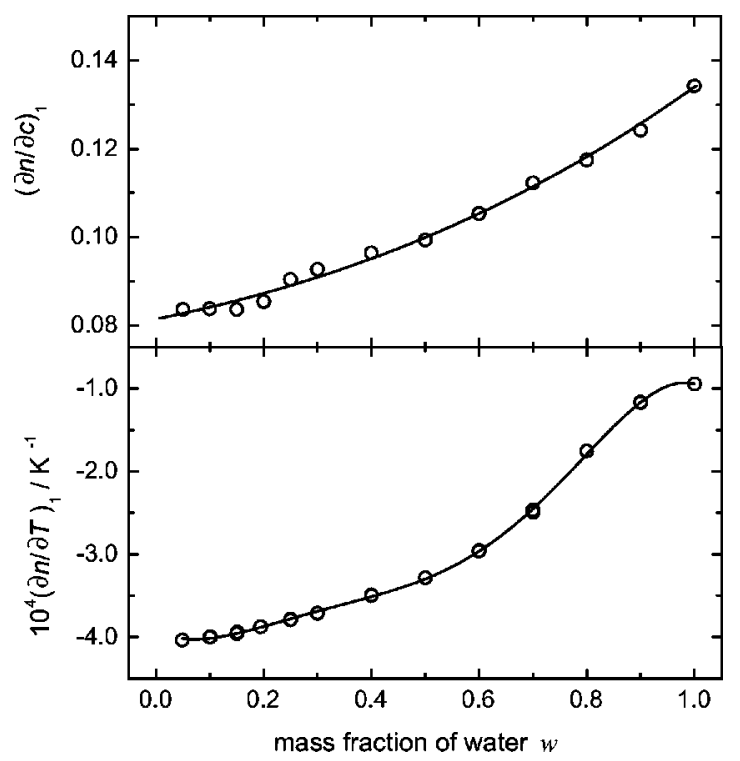

FIG. 2. $(\partial n / \partial c)_{1}$ and $(\partial n / \partial T)_{1}$ of $5 \mathrm{~g} / \mathrm{L}$ PEO in mixed water/ethanol solvent as a function of mass fraction of water for the water/ethanol mixture.

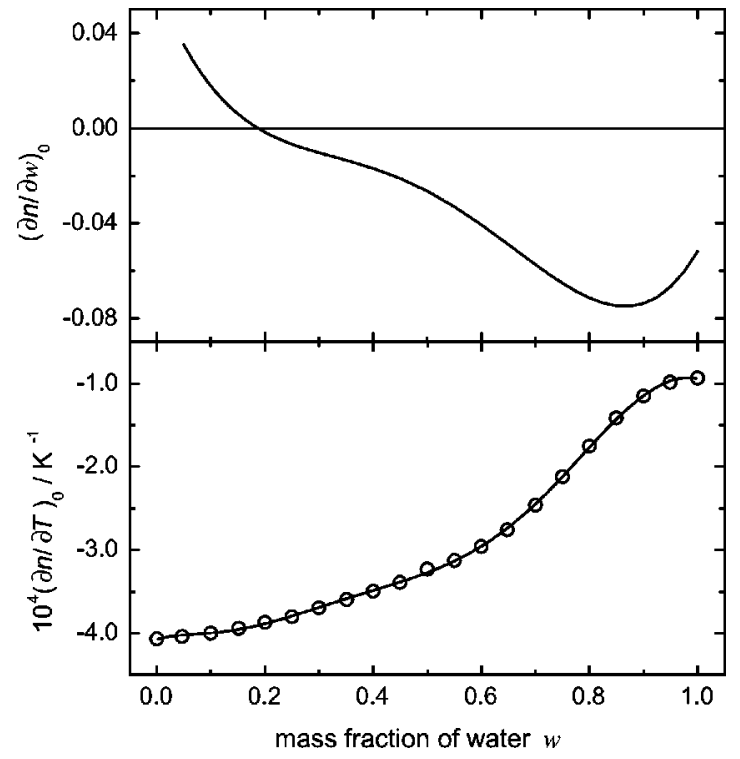

FIG. 3. $(\partial n / \partial w)_{0}$ and $(\partial n / \partial T)_{0}$ of water/ethanol as a function of mass fraction of water $w$.

The values of $(\partial n / \partial w)_{0}$ at $20^{\circ} \mathrm{C}$ represented by the solid line correspond to measurements with the sodium $D$ line as tabulated in the CRC handbook. ${ }^{39}$

Values for the contrast factors are presented in Tables I and II. For the PEO/water/ethanol system, $(\partial n / \partial c)_{1}$ does not depend strongly on water content for $w<0.2$ and increases with increasing water content for $w>0.2$. On the other hand, for water/ethanol mixtures, the sign of $(\partial n / \partial w)_{0}$ changes at $w=0.19$ from positive to negative with increasing water content.

\section{B. The binary system water/ethanol}

Figure 4 shows the Soret coefficient $S_{\mathrm{T}}$, diffusion coefficient $D$, and thermal diffusion coefficient $D_{\mathrm{T}}$ of water/ ethanol mixtures as a function of mass fraction of water $w$. Closed squares represent our experimental results obtained from a least squares fit to Eq. (2) of the normalized hetero-

TABLE I. Refractive index increment with respect to the mass fraction of PEO in water/ethanol, $(\partial n / \partial c)_{1}$, and refractive index increment with respect to the temperature $(\partial n / \partial T)_{1}$ of $5 \mathrm{~g} / \mathrm{L}$ PEO in water/ethanol. Here, $c$ corresponds to the mass fraction of PEO in the mixed solvent and $w$ corresponds to the mass fraction of water solvent.

\begin{tabular}{cccc}
\hline \hline$w$ & $(\partial n / \partial c)_{1}$ & $w$ & $10^{4}(\partial n / \partial T)_{1} / \mathrm{K}^{-1}$ \\
\hline 0.050 & 0.0836 & 0.049 & -4.0353 \\
0.100 & 0.0838 & 0.101 & -3.9983 \\
0.150 & 0.0836 & 0.150 & -3.9487 \\
0.200 & 0.0854 & 0.194 & -3.8751 \\
0.250 & 0.0904 & 0.250 & -3.7885 \\
0.300 & 0.0927 & 0.300 & -3.7121 \\
0.400 & 0.0964 & 0.400 & -3.4967 \\
0.500 & 0.0993 & 0.500 & -3.2834 \\
0.600 & 0.1053 & 0.600 & -2.9595 \\
0.700 & 0.1123 & 0.700 & -2.4804 \\
0.800 & 0.1175 & 0.800 & -1.7532 \\
0.900 & 0.1242 & 0.900 & -1.1650 \\
1 & 0.1341 & 1 & -0.9434 \\
\hline \hline
\end{tabular}


TABLE II. Refractive index increment with respect to temperature $(\partial n / \partial T)_{0}$ of water/ethanol mixtures. $w$ represents the mass fraction of water.

\begin{tabular}{cccc}
\hline \hline$w$ & $10^{4}(\partial n / \partial T)_{0} / \mathrm{K}^{-1}$ & $w$ & $10^{4}(\partial n / \partial T)_{0} / \mathrm{K}^{-1}$ \\
\hline 0 & -4.0688 & 0.5504 & -3.1284 \\
0.0468 & -4.0394 & 0.6000 & -2.9606 \\
0.1000 & -4.0015 & 0.6483 & -2.7585 \\
0.1514 & -3.9420 & 0.7000 & -2.4629 \\
0.2000 & -3.8707 & 0.7502 & -2.1226 \\
0.2499 & -3.8029 & 0.8000 & -1.7538 \\
0.3000 & -3.6962 & 0.8495 & -1.4167 \\
0.3511 & -3.5976 & 0.9002 & -1.1514 \\
0.4000 & -3.4954 & 0.9491 & -0.9889 \\
0.4499 & -3.3899 & 1 & -0.9378 \\
0.5000 & -3.2317 & & \\
\hline \hline
\end{tabular}

dyne signal obtained in our TDFRS experiments on water/ ethanol mixtures. For comparison, Fig. 4 includes experimental results reported by Kolodner et al. ${ }^{19}$ Zhang et al. ${ }^{20}$ and Dutrieux et al. ${ }^{21}$ which are restricted to mixtures of high water content. The figure shows that our experimental results on water/ethanol mixtures are in good agreement with the literature data. ${ }^{19-21,40} \mathrm{We}$ observe a sign change of $S_{\mathrm{T}}$ at $w$ $=0.71$, which agrees well with the work by Kolodner et al. ${ }^{19}$ and Zhang et al. ${ }^{20}$ Recent molecular dynamics simulations of the Soret effect in water/ethanol mixtures also agree with our results. ${ }^{23,24}$ The evaluation of TDFRS experiments on water/ ethanol mixtures is difficult for mixtures near $w=0.19$ since the contrast factor becomes very small. As Fig. 3 indicates,

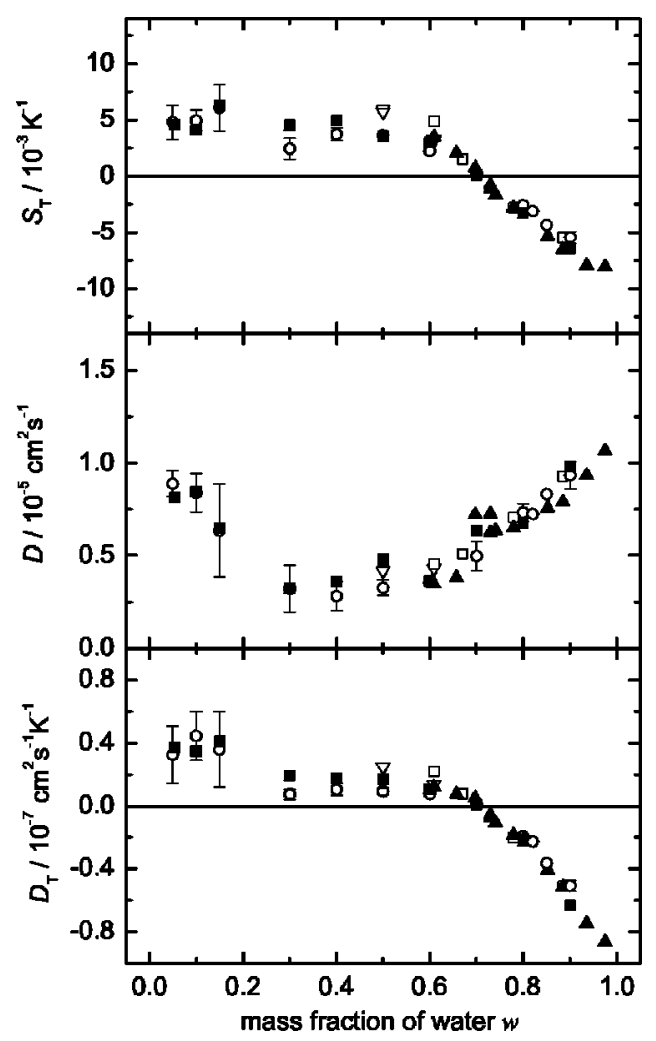

FIG. 4. Soret coefficient $S_{\mathrm{T}}$, diffusion coefficient $D$, and thermal diffusion coefficient $D_{\mathrm{T}}$ of water/ethanol as a function of mass fraction of water $w$ for binary system in this work $(\boldsymbol{\square})$, fast mode of ternary system in this work (○), Kolodner et al. at $20^{\circ} \mathrm{C}(\boldsymbol{\Delta})$, Zhang et al. at $25^{\circ} \mathrm{C}(\square)$, and Dutrieux et al. at $22.5^{\circ} \mathrm{C}(\nabla)$.

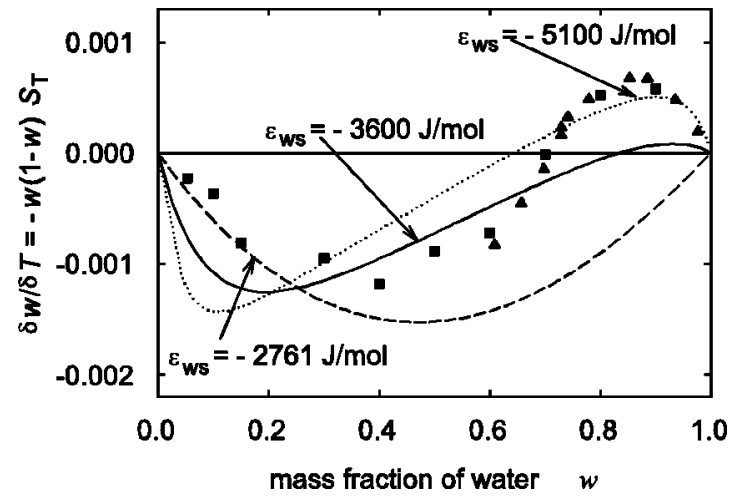

FIG. 5. Mass fraction differential $\delta w / \delta T=-w(1-w) S_{\mathrm{T}}$ of water in binary water/ethanol mixtures as a function of mass fraction $w$ of water. The symbols correspond to experimental data: this work (ם), Kolodner et al. at $20{ }^{\circ} \mathrm{C}(\boldsymbol{\Delta})$. The lines are calculated from the two-chamber lattice model at $20^{\circ} \mathrm{C}$ with mixed interaction parameters as indicated.

$(\partial n / \partial w)_{0}$ is zero at $w=0.19$ so that the modulation of the concentration does not lead to a modulation of the index of refraction, cf. Eq. (A8). This leads to a small signal-to-noise ratio of the heterodyne signal $\zeta_{\text {het }}(t)$ for lower water contents and is the origin of the relatively large errors in this regime.

As explained in more detail in Sec. IV C, the fast mode observed in our TDFRS experiments on the ternary system $\mathrm{PEO} /$ water/ethanol is associated with thermodiffusion properties of the solvent (water/ethanol). The open circles in Fig. 4 represent results obtained from the fast mode for the ternary system, where the error bars represent one standard deviation. In general, the results from measurements on binary and ternary mixtures agree well. As for the binary mixtures, small contrast factors lead to large uncertainties of the results for low water concentrations. This effect is also most likely the reason for the deviations between the values of the Soret coefficients of water determined from measurements on the binary and ternary systems at $w=0.3$ and 0.4 . In the experimentally investigated range the analysis of the fast mode measured for the ternary mixture give the same values (within experimental error) for $S_{\mathrm{T}}, D_{\mathrm{T}}$, and $D$ of water as obtained for the binary mixture. However, this behavior cannot persist for solvent mixtures very near the pure limit. For PEO in water, for example, the Soret coefficient of water has the same magnitude as the Soret coefficient of PEO. Hence, in the limit where the ethanol content goes to zero, the Soret coefficient of water has a value about 30 times its typical value in ethanol water mixtures. The analysis of the fast mode of the ternary mixture and the evaluation of the binary measurements revealed negative values of $S_{\mathrm{T}}$ of water in the range $w>0.71$. For the binary mixture, this corresponds to water molecules migrating to the hot side and ethanol molecules migrating to the cold side. We discuss the effect of the polymer on the thermodiffusion of the solvent in the following section.

In Fig. 5 we present values for the mass fraction differential $\delta w / \delta T=-w(1-w) S_{\mathrm{T}}$ of water in binary water/ ethanol mixtures. We compare experimental values (symbols) with results from the two-chamber lattice model ${ }^{35,36}$ for three different values of the interaction energy $\epsilon_{\mathrm{w}, \mathrm{s}}$ describing interactions between ethanol and water. In the lattice model 
for water/ethanol mixtures, sites of a simple cubic lattice are either occupied by water or ethanol or remain void, to account for compressibility. Interactions between occupied nearest neighbor sites are described by interaction energies and evaluated in random mixing approximation. Hydrogen bonding is not taken into account for water/ethanol mixtures. To determine Soret coefficients, two lattices at slightly different temperatures are considered and all possible lattice occupations consistent with a given total particle number are evaluated. The dashed line in Fig. 5 corresponds to the value $\epsilon_{\mathrm{w}, \mathrm{s}}=-2761 \mathrm{~J} / \mathrm{mol}$, i.e., the geometric mean of the waterwater and ethanol-ethanol interaction energies. As discussed in more detail in Ref. 35, this choice does not lead to a change in sign of the Soret coefficient of water. The value $\epsilon_{\mathrm{w}, \mathrm{s}}=-3600 \mathrm{~J} / \mathrm{mol}$ (solid line) was determined from a fit to tabulated density data, ${ }^{15,36}$ while $\epsilon_{\mathrm{w}, \mathrm{s}}=-5100 \mathrm{~J} / \mathrm{mol}$ was chosen to yield a good fit to the mass fraction differential at very high water concentrations. In agreement with the observations by Nieto-Draghi et al., ${ }^{23}$ we find that the mixed interaction has to be stronger than the interactions between the pure components in order to describe a sign change of the Soret coefficient with a simple lattice model. The comparison with experimental data shows that a single mixed interaction energy is not sufficient to describe the Soret effect over the whole concentration range. This is to be expected since our model does not currently take hydrogen bonding in water/ethanol mixtures into account. Figure 5 shows that the experimental values for the mass fraction differential vary most rapidly in the concentration range between $w=0.6$ and $w=0.8$. This is a concentration range of large structural changes in the liquid. ${ }^{41}$ In an NMR study, for example, it has been reported that the strength of hydrogen bonds formed among water molecules in pure water $(w=1)$ is enhanced by addition of ethanol up to $w=0.82$, whereas the hydrogenbond network is disrupted by further addition of ethanol and disappears around $w=0.61{ }^{42}$ Similarly, a dielectric relaxation measurement on water/ethanol showed large structural changes in the concentration range between $w=0.82$ and $w$ $=0.64 .{ }^{43}$ In summary, when ethanol is added to water, the hydrogen-bond network between water molecules remains intact up to a concentration of $18 \%$ ethanol by weight ( $w$ $=0.82$ ). Further addition of ethanol leads to the destruction of the hydrogen-bond network, which is complete at a mass fraction of about $w=0.6 .{ }^{41}$

\section{Ternary system of PEO/water/ethanol}

For the ternary system of $5 \mathrm{~g} / \mathrm{L}$ PEO in water/ethanol mixtures we present in Fig. 6 typical normalized heterodyne signal intensities as a function of time after the intensity grating has been switched on at time $t=0$. The three graphs correspond to three different solvent mixtures with mass fractions $w=0.5,0.8$, and 0.9 of water. The insets show the same data in semilogarithmic representation. The linear plots include data from the rapid increase of $\zeta_{\text {het }}(t)$ as the temperature modulation is established on the time scale $\tau_{\text {th }}$, which is less than $1 \mathrm{~ms}$. For later times, two modes are observed on typical time scales of $\tau_{1} \sim 10^{-3} \mathrm{~s}$ and $\tau_{2}$ $\sim 10^{-1} \mathrm{~s}$, respectively. This can be seen most clearly in the semilogarithmic plots of $\zeta_{\text {het }}(t)$, where the signal corre-
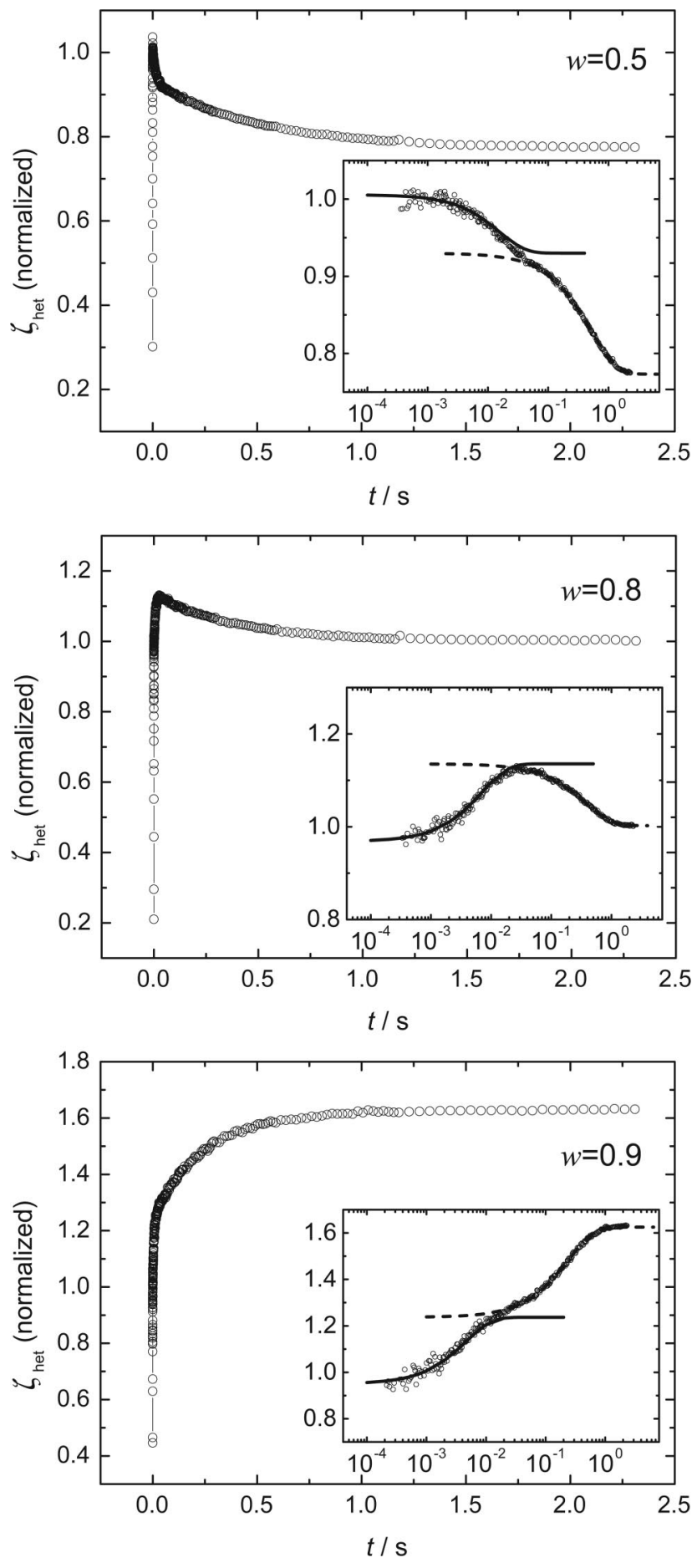

FIG. 6. Typical normalized TDFRS signals of $5 \mathrm{~g} / \mathrm{L}$ PEO/water/ethanol at solvent compositions $w=0.5,0.8$, and 0.9 . The insets show the semilogarithmic plots.

sponding to the establishment of the temperature gradient has been omitted for clarity. The solid and dashed lines represent the signal contributions of the fast and slow modes, respectively, and were obtained from a least squares fit to Eq. (3) for the normalized heterodyne signal.

The graphs of the heterodyne signals in Fig. 6 show two well separated modes. The slow mode reflects the growth of the concentration modulation of the polymer in the mixed solvent, while the fast mode reflects the growth of a concen- 


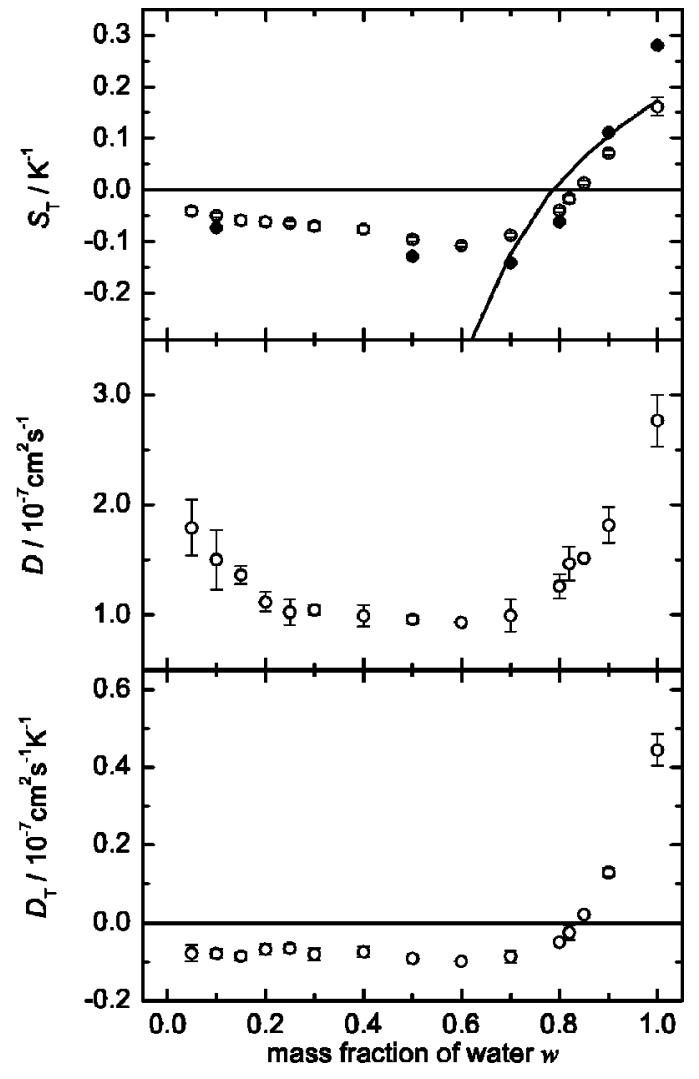

FIG. 7. Soret coefficient $S_{\mathrm{T}}$, diffusion coefficient $D$, and thermal diffusion coefficient $D_{\mathrm{T}}$ of PEO in water/ethanol as a function of water content of the solvent $w$. The open and closed symbols correspond to a polymer concentration of $5 \mathrm{~g} / \mathrm{L}$ and $1 \mathrm{~g} / \mathrm{L}$, respectively, the solid line represents values calculated from the two-chamber lattice model (Ref. 15).

tration modulation within the solvent mixture. As discussed in Sec. IV B, the analysis of the fast mode in experiments on the ternary system leads to results for the thermal and mass diffusion coefficients that agree with the results obtained from measurements on the binary system in the experimentally investigated range. For $w=0.5$ and 0.9 , the amplitudes of the signal contributions from the fast and the slow modes have the same sign, whereas for $w=0.8$ the amplitudes have opposite signs (the contribution of the fast mode increases with time while the contribution of the slow mode decreases with time). For the fast mode, the negative amplitude at $w$ $=0.5$ and the positive amplitudes at $w=0.8$ and 0.9 correspond to positive and negative values of the Soret coefficient of water, respectively. For the slow mode, the negative amplitudes at $w=0.5$ and 0.8 and the positive amplitude at $w$ $=0.9$ correspond to negative and positive values, respectively, of the Soret coefficient of PEO in the water/ethanol mixed solvent.

Values for the mass diffusion coefficients and the Soret coefficients were obtained for fast and slow modes from a fit of experimental heterodyne signals to Eq. (3). Transport coefficients for the solvents as obtained from the fast modes have already been presented in Fig. 4. In Fig. 7, we show results for the Soret coefficient $S_{\mathrm{T}}$, the mass diffusion coefficient $D$, and the thermal diffusion coefficient $D_{\mathrm{T}}=D S_{\mathrm{T}}$, for PEO in mixed water/ethanol solvent as obtained from the slow modes of the heterodyne signal. The thermal diffusion coefficient $D_{\mathrm{T}}$ of PEO is almost constant in the range $w$ $<0.7$ and increases with increasing water content for $w$ $>0.7$. Our value for $D_{\mathrm{T}}$ in pure water agrees well with the value $D_{\mathrm{T}}=5.5 \times 10^{-8} \mathrm{~cm}^{2} \mathrm{~s}^{-1} \mathrm{~K}^{-1}$ recently reported by Chan et al. ${ }^{44}$ for polyethylene glycol in water at $25^{\circ} \mathrm{C}$, where the $D_{\mathrm{T}}$ value was found to be independent of the molecular weight of polyethylene glycol. ${ }^{44}$

The diffusion coefficient $D$ of PEO as a function of water content of the solvent decreases for $w<0.25$, remains fairly constant for $0.25<w<0.7$, and increases for $0.7<w$. Comparing the variation of $D$ with the variation of the solvent viscosity we find that the self-diffusion coefficient is inversely proportional to the solvent viscosity for mixtures with higher water contents, $w>0.4$, and for mixtures with very low water contents, $w<0.15$. In the intermediation composition range, the diffusion coefficient is somewhat smaller than expected from the viscosity variation.

For PEO in pure water $(w=1.0)$ the Soret coefficient is positive in agreement with results from the literature. ${ }^{15,44,45}$ As the water content of the solvent decreases, the values of $S_{\mathrm{T}}$ decrease sharply and change sign at a composition of $w$ $=0.83$. The Soret coefficients continue to decrease with decreasing water content until they reach a minimum at $w$ $=0.6$. Upon further addition of ethanol, they increase slowly through the remaining concentration range. The solid line in the graph of Soret coefficients represents theoretical results from the two-chamber lattice model. ${ }^{15}$ The calculated values for $S_{\mathrm{T}}$ are positive for high water content of the solvent, decrease rapidly as the ethanol content is increased, change sign, and go through a minimum (not shown) in qualitative agreement with the experimental data. The lattice model treats short chains in exact enumeration and takes only hydrogen bonding between water molecules and the polymer into account. In order to achieve quantitative agreement between theory and experiment, the model has to be extended to include hydrogen bonding between solvent molecules and longer chains.

For lower water content $(w<0.25)$, the values of $S_{\mathrm{T}}$ are somewhat smaller than reported by us previously. ${ }^{16}$ In our earlier work on ethanol-rich mixtures, the heterodyne signal was fit to a single decay mode [cf. Eq. (2)] since the contributions from the fast mode are quite small and were assumed to be negligible. In this paper we evaluate the signal with a two-mode equation, Eq. (3), even when the the contribution of the fast mode is small. In this way, the amplitude of the slow-mode contribution is systematically smaller by $6 \%-$ $10 \%$ than the amplitude of the only mode evaluated in our previous work. ${ }^{16}$

Figure 7 includes experimental $S_{\mathrm{T}}$ data for two different polymer concentrations, $5 \mathrm{~g} / \mathrm{L}$ PEO and $1 \mathrm{~g} / \mathrm{L}$ PEO. For dilute solutions of PS in toluene, the Soret coefficient of PS was observed to decrease linearly with increasing concentration of PS. ${ }^{11,12}$ Our results follow a similar trend; the magnitude of the Soret coefficient is larger for the lower concentration of PEO for all solvent compositions, even where $S_{\mathrm{T}}$ is negative. The sign change of $S_{\mathrm{T}}$ appears to occur at the same composition of the solvent mixture, i.e., at $w=0.83$ for each sample. This behavior might change if we increase the PEO concentration further, so that PEO-PEO interactions become 


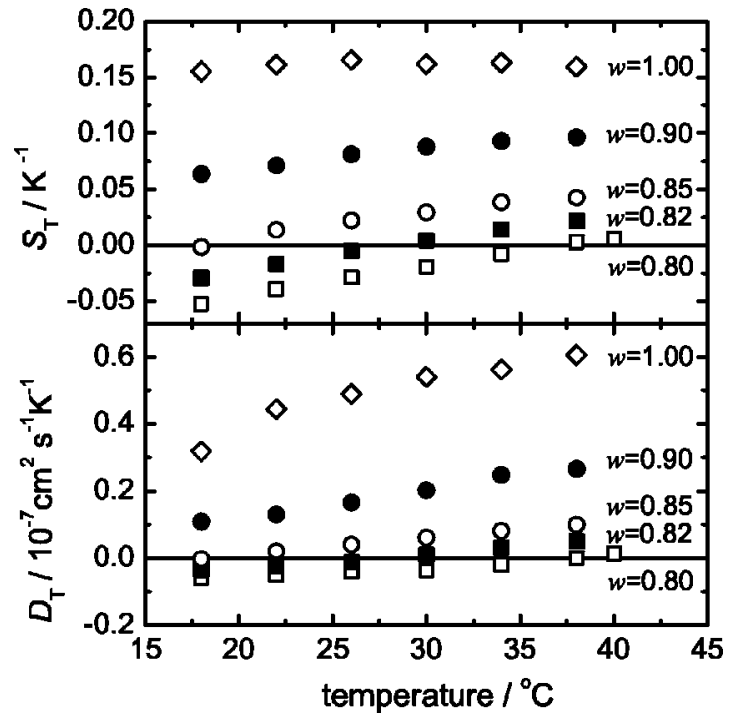

FIG. 8. Temperature dependence of $D_{\mathrm{T}}$ and $S_{\mathrm{T}}$ of $5 \mathrm{~g} / \mathrm{L}$ PEO in water/ ethanol for five mass fractions of water for water/ethanol, $w=0.80,0.82$, $0.85,0.90$, and 1.0 .

as important as the PEO-solvent interactions.

The temperature dependence of $D_{\mathrm{T}}$ and $S_{\mathrm{T}}$ for a polymer concentration of $5 \mathrm{~g} / \mathrm{L}$ PEO has been investigated for five different solvent compositions. The results presented in Fig. 8 show an increase of $D_{\mathrm{T}}$ and $S_{\mathrm{T}}$ with increasing temperature for all compositions measured. As the water concentration of the solvent increases, the slope of $S_{\mathrm{T}}$ as a function of temperature decreases, while the slope of $D_{\mathrm{T}}$ increases. The larger temperature dependence of $D_{\mathrm{T}}$ in water reflects an increase in the mass diffusion coefficient $D$, which is related to a decrease in the viscosity. A sign change of $D_{\mathrm{T}}$ and $S_{\mathrm{T}}$ is observed for the compositions $w=0.80,0.82$, and 0.85 , where the temperature at which the sign change occurs decreases with increasing water content. For very high water content, $w=0.9$ and 1.0, a sign change has not observed in the temperature range of the experiment. Lattice model calculations also predict sign changes of the Soret coefficients with temperature. ${ }^{35}$ Unfortunately, the experimentally observed positive slope of $S_{\mathrm{T}}$ as a function of temperature is realized in the model only for lower water contents in the temperature range of the experiments. We hope that an extended model, as discussed above, will resolve this discrepancy and also lead to a better understanding of the change in temperature dependence with solvent composition. In addition, experiments that probe the solvent quality, such as light scattering studies to determine the radius of gyration and the second virial coefficient for the PEO/water/ethanol system, may lead to a better understanding of the temperature dependence of the Soret coefficient.

\section{Discussion}

In our earlier work we discussed in detail the correlation between solvent quality and thermal diffusion. ${ }^{15,35}$ In general, we expect the Soret coefficient to be positive for a polymer in good solvent, and we expect $S_{\mathrm{T}}$ to decrease, as the solvent becomes poorer. This agrees qualitatively with the

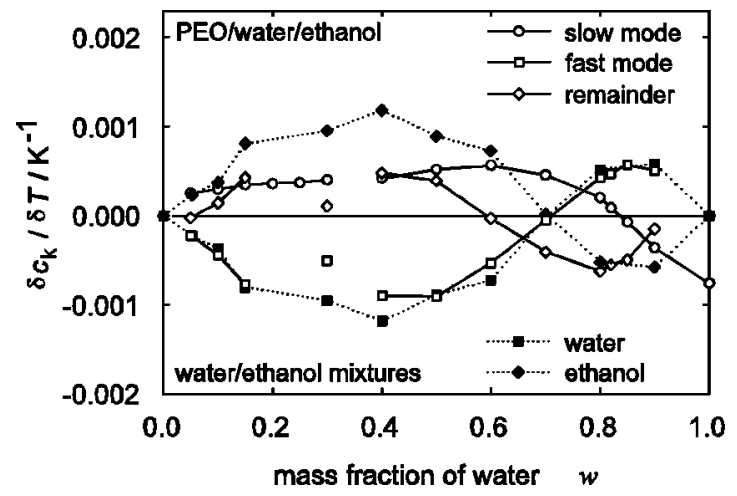

FIG. 9. Mass fraction differentials $\delta c_{k} / \delta T$ of the binary mixtures water/ ethanol, $c_{k} \in\{w,(1-w)\}$, and the ternary mixtures PEO/water/ethanol, $c_{k}$ $\in\{c, w,(1-c-w)\}$, as a function of solvent composition. Closed and open symbols represent data for the binary and ternary systems, respectively. The lines are guides to the eye.

behavior observed for PEO in water/ethanol solutions, since water is an excellent solvent for PEO while ethanol is a poor solvent.

In order to investigate further the effect of solvent composition on thermodiffusion of the polymer we present in Fig. 9 the Soret coefficient data for both binary and ternary systems in the form of mass fraction differentials. The differentials are calculated from the Soret coefficients according to $\delta c_{k} / \delta T=-c_{k}\left(1-c_{k}\right) S_{k T}$, cf. Eq. (4). They have the opposite sign of the corresponding Soret coefficients and hence are positive if the corresponding component is enriched in the warmer regions of the fluid. For the ternary system, the slow mode is associated with the polymer, the fast mode is associated with the solvent. The "remainder" is obtained from the condition that the three concentration differences add up to zero, $\delta c+\delta w+\delta s=0$, where $c, w$, and $s=1-c$ $-w$ are the mass fractions of the polymer, water, and ethanol, respectively. For our system, the fast mode and the remainder turn out to be related to the water and ethanol signals, respectively (see below). The differentials change sign at the same solvent composition as the corresponding Soret coefficients, i.e., $w=0.83$ for the polymer and $w=0.71$ for the binary system and the fast mode in the ternary system. Furthermore, the "remainder" of the ternary system changes sign at $w=0.59$. The data suggest to differentiate three concentration regimes, region I, $w<0.59$, region II, $0.59<w$ $<0.83$, and region III, $w>0.83$. For ternary solutions with high water content, $w>0.83$, PEO and the remainder are enriched in cooler regions of the fluid, just as ethanol is for binary mixtures. Conversely, for low water contents, $w$ $<0.59$, PEO and the remainder as well as ethanol in binary mixtures are enriched in warmer regions. In the intermediate composition range, $0.83>w>0.59$, the polymer and the remainder do not have the same thermodiffusive behavior but migrate to warm and cold regions, respectively. Furthermore, in the center of region II, the Soret coefficient of water changes sign. A comparison of the boundaries of region II with the concentration range, $0.6<w<0.82$, where large structural changes take place in binary ethanol water mixtures $^{41-43}$ (see Sec. IV B), suggests that the formation of hydrogen bonds plays a major role in the Soret effect. 
The data for the fast mode in ternary mixtures are seen to follow closely those of water in binary solutions. While this cannot be true for all concentrations (the fast mode vanishes as $w \rightarrow 1$ ) preliminary calculations with the twochamber lattice model show that the deviations become large only for $w>0.9$. Hence, we tentatively identify the fast mode of the ternary system with a water signal and the "remainder" with ethanol. Under this assumption, it appears that the thermodiffusive behavior of water molecules is not sensitive to a replacement of ethanol molecules by a PEO chain. This is not unreasonable given that water forms hydrogen bonds with both PEO and ethanol.

In earlier work, ${ }^{15,35,36}$ we related the Soret effect of a polymer in dilute solution to the internal energy difference of two chambers at equal temperatures, separated by a semipermeable membrane that allows only solvent molecules to move freely between the chambers. If a chamber containing only solvent molecules has a lower internal energy than a chamber containing polymer and solvent, then the polymer will be enriched in the warmer regions when the fluid is subject to a temperature gradient. Applied to the intermediate concentration region II, this suggests that the replacement of ethanol molecules by a PEO chain leads to an increase in the internal energy. Since hydrogen bonds lower the internal energy contribution of a molecule dramatically, one may conclude that ethanol is more effective in forming hydrogen bonds with water than PEO, in region II.

Iacopini and Piazza ${ }^{17}$ recently investigated thermophoresis in protein (lysozyme) solutions and found the Soret coefficient to change from negative to positive with increasing temperature. Hence, the protein is enriched in warmer regions of the fluid at lower temperatures but migrates to the cold side at higher temperatures, just as PEO in mixed ethanol/water solvent. While the lysozyme solutions, buffered and with added $\mathrm{NaCl}$, are very complex systems, the similarity in the temperature dependence of the Soret coefficient suggests that similar factors determine thermodiffusion in the protein solutions and the PEO/ethanol/water system. In both systems, hydrogen bonds play an important role. Furthermore, Iacopini and Piazza correlated the temperature variation of the Soret coefficient with the solubility of the protein and found that an increase in solubility corresponds to an increase in the Soret coefficient. This agrees with our earlier work, ${ }^{15,35}$ where we found that negative Soret coefficients for a polymer are associated with marginal solvents while an increase in solvent quality leads to an increase in the Soret coefficient.

From previous observations that the thermal diffusion coefficient $D_{\mathrm{T}}$ is independent of the molecular weight for dimers, trimers, and chains up to several hundred repeat units ${ }^{46-48}$ we conclude that the thermal diffusion process takes place on short length scales, which may be comparable to the length scale $\left(\sim 10^{-10} \mathrm{~m}\right)$ of heat transport. This suggests that the sign change of the system can also be observed experimentally for short PEO chains, which are also more easily investigated by computer simulations. Furthermore, we expect that the first solvation shell of the PEO molecules determines the thermodiffusive behavior. This agrees with our observation that the Soret coefficients of all three com- ponents change sign in the composition range where water/ ethanol mixtures undergo large structural changes.

\section{CONCLUSION}

The Ludwig-Soret effect for ternary PEO/water/ethanol and binary water/ethanol mixtures was studied by TDFRS. For the ternary system, the signal decays with two decay times corresponding to the establishment of a concentration gradient of the solvent followed by the establishment of the concentration gradient of the polymer in the solvent mixture. The sign of the Soret coefficient of PEO is positive in the solvent composition range $w>0.83$, i.e., the polymer migrates to the colder regions of the fluid as is typical for polymer solutions. In contrast, the sign of $S_{\mathrm{T}}$ is negative in the range $w<0.83$, i.e., the polymer migrates to the warmer regions of the fluid, which is very unusual. A comparison of thermodiffusion in the binary and ternary systems shows that the largest changes in the Soret coefficients occur in both cases in the concentration range, where water/ethanol mixtures undergo large structural changes. Hydrogen-bond formation plays a major role both in the solubility of PEO in water/ethanol solutions and in the thermodiffusion of PEO.

\section{ACKNOWLEDGMENTS}

The authors are very much indebted to Gerhard Wegner for his support of the work and for valuable discussions. Beate Müller helped with the measurements of the refractive index increments. We thank BASF for the Basantol Gelb 215. Financial support through the National Science Foundation (Grant No. DMR-0103704), the Petroleum Research Fund (Grant No. 36559-GB7), the Research Corporation (Grant No. CC5228), and the Fonds der Chemischen Industrie is gratefully acknowledged.

\section{APPENDIX: DERIVATION OF THE WORKING EQUATIONS}

The diffusive transport of heat and matter in a fluid mixture of $K$ components is described by $K$ flows, a heat flow, and $K-1$ independent mass flows. We work in the volumefixed reference frame and denote the mass flows by $\mathbf{J}_{j}^{0}, j$ $\in\{1, \ldots, K\}$. The flow of component $K$ is related to the other flows by $v_{K} \mathbf{J}_{K}^{0}=-\sum_{j=1}^{K-1} v_{j} \mathbf{J}_{j}^{0}$, where $v_{j}$ is the partial specific volume of component $j$. If the mean volume velocity can be neglected, as is typical for liquid mixtures, ${ }^{5}$ the law of mass conservation can be expressed as

$$
\frac{\partial \rho_{k}}{\partial t}=-\nabla \cdot \mathbf{J}_{k}^{0},
$$

where $\rho_{k}$ is the mass density of component $k$. Diffusion coefficients are defined through the phenomenological equations

$$
\mathbf{J}_{k}^{0}=-\sum_{j=1}^{K-1} D_{k j} \boldsymbol{\nabla} \rho_{j}-D_{k T}^{\prime} \boldsymbol{\nabla} T .
$$

The $(K-1)^{2}$ mass diffusion coefficients $D_{k j}$ are complicated combinations of the Onsager mass transport coefficients and thermodynamic derivatives. ${ }^{5}$ Due to Onsager's reciprocal re- 
lations, only $K(K-1) / 2$ of these diffusion coefficients are independent. The prime on the symbol $D_{\mathrm{kT}}^{\prime}$ indicates that $D_{\mathrm{kT}}^{\prime}$ is a nonstandard form of the thermal diffusion coefficients, which we employ temporarily. Inserting the current (A2) into Eq. (A1) for the mass flow, we obtain the thermodiffusion equations

$$
\frac{\partial \rho_{k}}{\partial t}=\sum_{j=1}^{K-1} D_{k j} \nabla^{2} \rho_{j}+D_{k T}^{\prime} \nabla^{2} T .
$$

Setting the flows to zero in Eq. (A2) we obtain for the ratio of mass fraction and temperature gradients in the volumefixed reference frame

$$
\frac{\boldsymbol{\nabla} c_{k}}{\boldsymbol{\nabla} T}=-\sum_{j, m=1}^{K-1}\left(\frac{\partial c_{k}}{\partial \rho_{m}}\right)_{T, P,\left\{\rho_{i \neq m}\right\}}\left(D^{-1}\right)_{m j} D_{j T}^{\prime},
$$

where the mass fraction $c_{k}$ of component $k$ is given by $c_{k}$ $=\rho_{k} / \rho$, with $\rho$ the mass density of the liquid, where $P$ is the pressure, and where $\left(D^{-1}\right)$ is the inverse of the matrix of mass diffusion coefficients $D_{k j}$.

In a TDFRS experiment, two interfering laser beams create an intensity grating, which is converted into a temperature grating through the absorption of energy by a trace amount of dye. Since the effect of concentration gradients on the temperature profile (Dufour effect) is small in liquid mixtures, ${ }^{5}$ the temperature as a function of position and time can be calculated from a heat equation. In the experiments, the time variation of the gratings is described by a series of step functions (on/off) in time. We will focus on the signal generated after the grating has been switched on at time $t$ $=0$. In this case, the spatial modulation of the temperature for times $t>0$ can be described by ${ }^{37}$

$$
T_{q}(t) e^{i q x} \equiv \Theta_{0}\left(1-e^{-t / \tau_{\mathrm{th}}}\right) e^{i q x},
$$

where $q$ is the wave number of the grating and the optical axis is parallel to the $x$ axis. The amplitude $\Theta_{0}$ is determined by the intensity of the laser beams, the absorption coefficient of the dye, and the heat capacity and thermal diffusivity of the liquid. The time constant of the temperature grating $\tau_{\text {th }}$ is related to the thermal diffusivity through $\tau_{\text {th }}=1 /\left(q^{2} D_{\text {th }}\right)$.

The spatial variation of the temperature induces mass flows through the Ludwig-Soret effect. The resulting spatial modulation of the composition may be represented as

$$
\delta c_{k}=c_{k}(x, t)-c_{k 0}=c_{k q}(t) e^{i q x},
$$

where $\delta c_{k}$ is the deviation of the mass fraction $c_{k}$ of component $k$ from its equilibrium value $c_{k 0}$. Both temperature and composition modulation lead to a spatial modulation of the index of refraction, which is detected by Bragg refraction of an additional laser.

$$
\delta n=n(x, t)-n_{0}=n_{q}(t) e^{i q x},
$$

where $\delta n$ is the deviation of the index of refraction $n$ from its equilibrium value $n_{0}$. The heterodyne signal intensity of the read out laser is proportional to the amplitude $n_{q}(t)$ of the refractive index modulation,

$$
n_{q}(t)=\left(\frac{\partial n}{\partial T}\right)_{P,\left\{c_{j}\right\}} T_{q}(t)+\sum_{k=1}^{K-1}\left(\frac{\partial n}{\partial c_{k}}\right)_{P, T,\left\{c_{j \neq k}\right\}} c_{k q}(t) .
$$

The contribution of the temperature modulation to $n_{q}(t)$ has a much smaller time constant $\left(\tau_{\text {th }}\right)$ than the concentration modulation. It is customary, to take the long time limit of this contribution, using the temperature modulation of Eq. (A5)

$$
n_{q}^{\mathrm{th}}=\lim _{t \rightarrow \infty} T_{q}(t)\left(\frac{\partial n}{\partial T}\right)_{P,\left\{c_{j}\right\}}=\Theta_{0}\left(\frac{\partial n}{\partial T}\right)_{P,\left\{c_{j}\right\}}
$$

and evaluate the normalized heterodyne signal

$$
\zeta_{\text {het }}=\frac{n_{q}(t)}{n_{q}^{\text {th }}} .
$$

In order to describe TDFRS experiments for binary mixtures, $K=2$, we solve the diffusion equation for the mass density of component 1 as obtained from Eq. (A3) for the geometry of the experiment

$$
\frac{\partial \rho_{1}}{\partial t}=D \frac{\partial^{2} \rho_{1}}{\partial x^{2}}+D_{1 T}^{\prime} \frac{\partial^{2} T}{\partial x^{2}}
$$

where we have dropped the subscripts on the mutual diffusion coefficient $D$. The expression for the temperature, Eq. (A5), suggests a solution for $\rho_{1}(x, t)$ of the form ${ }^{49}$

$$
\rho_{1}(x, t)=\rho_{10}+\rho_{1 q}(t) e^{i q x},
$$

where $\rho_{10}$ is the equilibrium value of $\rho_{1}$. Solving Eq. (A11) with this ansatz and Eq. (A5) we find

$$
\rho_{1 q}(t)=-\frac{\Theta_{0} D_{1 T}^{\prime}}{\left(\tau-\tau_{\mathrm{th}}\right) D}\left\{\tau\left(1-e^{-t / \tau}\right)-\tau_{\mathrm{th}}\left(1-e^{-t / \tau_{\mathrm{th}}}\right)\right\},
$$

for times $t>0$, where $\tau=1 /\left(q^{2} D\right)$ is the time constant of mutual diffusion. In a typical TDFRS experiment, the lifetime of the temperature grating $\tau_{\text {th }}$ is much smaller than the time constant of diffusion, $\tau_{\mathrm{th}} \ll \tau$. With this approximation, the mass density modulation $\rho_{1 q}$ simplifies to

$$
\rho_{1 q}(t)=-\Theta_{0} \frac{D_{1 T}^{\prime}}{D}\left(1-e^{-t / \tau}\right) .
$$

In order to obtain the modulation of the mass fraction $c_{1}$ of component 1 , which is used in the evaluation of the heterodyne signal, we insert the modulation of the mass density, Eq. (A14), into the relation

$$
c_{1 q}(t)=\left(\frac{\partial c_{1}}{\partial \rho_{1}}\right)_{T, P} \rho_{1 q}(t)
$$

and obtain

$$
c_{1 q}(t)=-\Theta_{0} c_{10}\left(1-c_{10}\right) S_{1 T}\left(1-e^{-t / \tau}\right),
$$

where, the Soret coefficient $S_{1 T}$ has been identified from Eqs. (4) and (A4) applied to binary mixtures $(K=2)$. Introducing the usual thermal diffusion coefficient $D_{T}$ through

$$
D_{T}=\frac{1}{c_{10}\left(1-c_{10}\right)}\left(\frac{\partial c_{1}}{\partial \rho_{1}}\right)_{T, P} D_{1 T}^{\prime},
$$


we recover the relation between the Soret coefficient and the thermal and mutual diffusion coefficients

$$
S_{\mathrm{T}} \equiv S_{1 T}=\frac{D_{\mathrm{T}}}{D} .
$$

Finally, the normalized heterodyne signal intensity for a binary mixture, as presented in Eq. (2), is obtained by inserting Eq. (A16) into Eq. (A8) for $K=2$ and evaluating Eq. (A10) with Eqs. (A9) and (A8).

For a ternary mixture, there are two independent mass flows. For the geometry and temperature distribution of the TDFRS experiment, Eqs. (A3) take the form

$$
\begin{aligned}
& \frac{\partial \rho_{1}}{\partial t}=D_{11} \frac{\partial^{2} \rho_{1}}{\partial x^{2}}+D_{12} \frac{\partial^{2} \rho_{2}}{\partial x^{2}}+D_{1 T}^{\prime} \frac{\partial^{2} T}{\partial x^{2}}, \\
& \frac{\partial \rho_{2}}{\partial t}=D_{21} \frac{\partial^{2} \rho_{1}}{\partial x^{2}}+D_{22} \frac{\partial^{2} \rho_{2}}{\partial x^{2}}+D_{2 T}^{\prime} \frac{\partial^{2} T}{\partial x^{2}} .
\end{aligned}
$$

As for the binary mixture, we look for solutions in the form

$$
\rho_{k}(x, t)=\rho_{k 0}+\rho_{k q}(t) e^{i q x}, \quad k \in\{1,2\},
$$

where $\rho_{k 0}$ is the equilibrium values of $\rho_{k}$. An investigation of the solutions of the coupled differential equations (A19) and (A20) shows that the modes decouple completely if the diffusion coefficients satisfy

$$
\left|D_{12}\right|,\left|D_{21}\right| \ll\left|D_{11}-D_{22}\right| \text {. }
$$

This condition is expected to hold for a polymer in mixed solvent, since (a) the off-diagonal elements $D_{i k}, i \neq k$ of the diffusion coefficient matrix are typically much smaller than the diagonal elements $D_{i i},{ }^{50}$ and (b) the diffusion coefficient of a polymer $\left(D_{22}\right)$ is typically much smaller than the diffusion coefficient of small-molecules fluids. Under the assumption that the off-diagonal diffusion coefficients can be neglected, the solution to Eq. (A19) is given by

$$
\rho_{k q}(t) \simeq-\Theta_{0} \frac{D_{k T}^{\prime}}{D_{k k}}\left(1-e^{-t / \tau_{k k}}\right), \quad k \in\{1,2\},
$$

where $\tau_{k k}=1 /\left(q^{2} D_{k k}\right)$, and where we have assumed again that the time constant of the thermal grating is much smaller than the time constants of diffusion, $\tau_{\text {th }} \ll \tau_{k k}$.

In the transformation from the mass density to the mass fraction modulation, mixed partial derivatives of the form $\left(\partial c_{k} / \partial \rho_{j}\right)_{T, P, \rho_{k}}, k \neq j$ are encountered. One of these derivatives is proportional to the mass fraction of the polymer, which is very small. The other depends on the composition of the solvent, but also does not contribute significantly for our solvent system. Hence, we neglect the mixed derivatives

$$
\left(\frac{\partial c_{k}}{\partial \rho_{j}}\right)_{T, P, c_{m \neq k}} \simeq 0, \quad k \neq j ; k, j, m \in\{1,2\},
$$

and use the approximation

$$
c_{k q} \simeq\left(\frac{\partial c_{k}}{\partial \rho_{k}}\right)_{T, P, c_{j \neq k}} \rho_{k q} .
$$

With the assumptions (A22) and (A24), both the matrix of mass diffusion coefficients and the matrix of partial derivatives of mass fractions with respect to mass densities become diagonal. In this case, Eqs. (4) and (A4) applied to ternary mixtures $(K=3)$ yield for the Soret coefficients

$$
S_{k T} \simeq \frac{1}{c_{k 0}\left(1-c_{k 0}\right)}\left(\frac{\partial c_{k}}{\partial \rho_{k}}\right)_{T, P, c_{j \neq k}} \frac{D_{k T}^{\prime}}{D_{k k}} .
$$

Thus, if conditions (A22) and (A24) are satisfied, the Soret coefficients of the ternary mixture are simply related to the thermal and mutual diffusion coefficients through

$$
S_{k T} \simeq \frac{D_{k \mathrm{~T}}}{D_{k k}},
$$

where, in analogy with Eq. (A17) for binary mixtures, we have defined the following unprimed thermal diffusion coefficients:

$$
D_{k T}=\frac{1}{c_{k 0}\left(1-c_{k 0}\right)}\left(\frac{\partial c_{k}}{\partial \rho_{k}}\right)_{T, P, c_{j \neq k}} D_{k T}^{\prime} .
$$

With Eqs. (A25) and (A26), the amplitudes of the mass fraction modulations in a ternary mixture have the simple form

$$
c_{k q}(t)=-\Theta_{0} c_{k 0}\left(1-c_{k 0}\right) S_{k T}\left(1-e^{-t / \tau_{k k}}\right) .
$$

Finally, the normalized heterodyne signal intensity for a ternary mixture, as presented in Eq. (3), is obtained by inserting Eq. (A29) into Eq. (A8) for $K=3$ and evaluating Eq. (A10) with Eqs. (A9) and (A8).

${ }^{1}$ C. Ludwig, Sitzungsber. Preuss. Akad. Wiss., Phys. Math. K1. 20, 539 (1856)

${ }^{2}$ Ch. Soret, Arch. Sci. Phys. Nat. 3, 48 (1879); C. R. Acad. Sci. III 91, 289 (1880); Ann. Chim. Phys. 22, 239 (1881)

${ }^{3}$ We use the following sign convention for $S_{\mathrm{T}}$ and $D_{\mathrm{T}}$ : In a binary mixture of $A$ and $B, S_{\mathrm{T}}$ of $A$ (the first named component) is positive, if $A$ moves to the cold side. This implies that $S_{\mathrm{T}}$ of $B$ is negative, because $B$ migrates to the warm side. The same holds for $D_{\mathrm{T}}$. This definition is independent of the density of the two components.

${ }^{4}$ W. Köhler and S. Wiegand, Thermal Non-equilibrium Phenomena in Fluid Mixtures (Springer, Berlin, 2002).

${ }^{5}$ S. R. de Groot and P. Mazur, Non-equilibrium Thermodynamics (Dover, New York, 1984).

${ }^{6}$ K. E. Grew and T. L. Ibbs, Thermal Diffusion in Gases (Cambridge University Press, Cambridge, 1952).

${ }^{7}$ J. J. Hurly and M. R. Moldover, J. Res. Natl. Inst. Stand. Technol. 105, 667 (2000).

${ }^{8}$ T. G. Heijmen, P. E. S. Wormer, P. E. S. , A. van der Avoird, R. E. Miller, and R. Moszynski, J. Chem. Phys. 110, 5639 (1999).

${ }^{9}$ G. Meyerhoff and K. Nachtigall, J. Polym. Sci. 57, 227 (1962).

${ }^{10}$ O. Ecenarro, J. A. Madariaga, J. L. Navarro, C. M. Santamaria, J. A. Carrion, and J. M. Saviron, Macromolecules 27, 4968 (1994).

${ }^{11}$ W. Köhler, C. Rosenauer, and P. Rossmanith, Int. J. Thermophys. 16, 11 (1995)

${ }^{12}$ K. J. Zhang, M. E. Briggs, R. W. Gammon, J. V. Sengers, and J. F. Douglas, J. Chem. Phys. 111, 2270 (1999).

${ }^{13}$ J. Rauch and W. Köhler, Phys. Rev. Lett. 88, 185901 (2002).

${ }^{14}$ M. Giglio and A. Vendramini, Phys. Rev. Lett. 38, 26 (1977).

${ }^{15}$ B. J. de Gans, R. Kita, S. Wiegand, and J. Luettmer-Strathmann, Phys. Rev. Lett. 91, 245501 (2003)

${ }^{16}$ B. J. de Gans, R. Kita, B. Müller, and S. Wiegand, J. Chem. Phys. 118, 8073 (2003)

${ }^{17}$ S. Iacopini and R. Piazza, Europhys. Lett. 63, 247 (2003).

${ }^{18}$ I. Prigogine, L. de Brouckère, and R. Amand, Physica (Amsterdam) 16, 851 (1950).

${ }^{19}$ P. Kolodner, H. Williams, and C. Moe, J. Chem. Phys. 88, 6512 (1988).

${ }^{20}$ K. J. Zhang, M. E. Briggs, R. W. Gammon, and J. V. Sengers, J. Chem. Phys. 104, 6881 (1996). 
${ }^{21}$ J. F. Dutrieux, J. K. Platten, G. Chavepeyer, and M. M. Bou-Ali, J. Phys. Chem. B 106, 6104 (2002).

${ }^{22}$ C. Debuschewitz and W. Köhler, Phys. Rev. Lett. 87, 055901 (2001).

${ }^{23}$ C. E. Nieto-Draghi, PhD thesis, Universitat Rovira i Virgili, 2003.

${ }^{24}$ B. Rousseau, C. E. Nieto-Draghi, and J. Bonet Ávalos, Phys. Rev. Lett. (to be published).

${ }^{25}$ J. E. Powers, in New Chemical Engineering Separation Techniques, edited by H. M. Schoen (Interscience, New York, 1962) Chap. 1, pp. 1-98.

${ }^{26}$ E. Bringuier and A. Bourdon, Phys. Rev. E 67, 011404 (2003); Phys. Rev. Lett. 87, 055901 (2001)

${ }^{27}$ J. K. G. Dhont, J. Chem. Phys. 120, 1632 (2004).

${ }^{28}$ J. K. G. Dhont, J. Chem. Phys. 120, 1642 (2004).

${ }^{29}$ A. C. Van Asten, W. Th. Kok, R. Tijssen, and H. Poppe, J. Polym. Sci., Part B: Polym. Phys. 34, 283 (1996).

${ }^{30}$ L. J. T. M. Kempers, J. Chem. Phys. 115, 6330 (2001)

${ }^{31}$ L. J. T. M. Kempers, J. Chem. Phys. 90, 6541 (1989).

${ }^{32}$ A. Firoozababi, K. Ghorayeb, and K. Shukla, AIChE J. 46, 892 (2000).

${ }^{33}$ J. P. Larre, J. K. Platten, and G. Chavepeyer, Int. J. Heat Mass Transfer 40, 545 (1997).

${ }^{34}$ C. A. Rue and M. E. Schimpf, Anal. Chem. 66, 4054 (1994).

${ }^{35}$ J. Luettmer-Strathmann, J. Chem. Phys. 119, 2892 (2003).

${ }^{36}$ J. Luettmer-Strathmann, Int. J. Thermophys. (to be published).
${ }^{37}$ A. Perronace, C. Leppla, F. Leroy, B. Rousseau, and S. Wiegand, J. Chem. Phys. 116, 3718 (2002).

${ }^{38}$ A. Becker, W. Köhler, and B. Müller, Ber. Bunsenges. Phys. Chem. 99, 600 (1995).

${ }^{39}$ The CRC Handbook of Chemistry and Physics, edited by D. R. Lide (CRC, Boca Raton, 1995).

${ }^{40}$ W. Köhler and B. Müller, J. Chem. Phys. 103, 4367 (1995).

${ }^{41}$ V. P. Belousov and M. Yu. Panov Thermodynamic Properties of Aqueous Solutions of Organic Substances, (CRC, Boca Raton, FL, 1994).

${ }^{42}$ A. Coccia, P. L. Indovina, F. Podo, and V. Viti, Chem. Phys. 7, 30 (1975).

${ }^{43}$ T. Sato, A. Chiba, and R. Nozaki, J. Chem. Phys. 110, 2508 (1999).

${ }^{44}$ J. Chan, J. J. Popv, S. Kolisnek-Kehl, and D. G. Leaist, J. Solution Chem. 32, 197 (2003).

${ }^{45}$ J. J. Kirkland and W. W. Yau, J. Chromatogr. 353, 95 (1986).

${ }^{46}$ M. E. Schimpf and J. C. Giddings, J. Polym. Sci., Part B: Polym. Phys. 27, 1317 (1989).

${ }^{47}$ P. Rossmanith and W. Köhler, Macromolecules 29, 3203 (1996).

${ }^{48}$ S. Wiegand, J. Phys.: Condens. Matter 16, R357 (2004).

${ }^{49}$ W. Köhler and R. Schäfer, Adv. Polym. Sci. 151, 1 (2000).

${ }^{50}$ H. J. V. Tyrrell and K. R. Harris, Diffusion in Liquids (Butterworths, London, 1984). 